\title{
Componentes naturales para el control de antracnosis (Colletotrichum gloeosporoides) en guanabana (Annona muricata L.)
}

\section{Natural components for the control of anthracnose (Colletotrichum gloeosporioides) in soursop (Annona muricata L.)}

\author{
Ladino Manjarrés Diego Alexander ${ }^{1}$ y Bastidas López Harold ${ }^{2}$ \\ ${ }^{1}$ Ing. Agrónomo, Universidad de los Llanos, e \\ ${ }^{2}$ Ing. Agrónomo, MSc. Docente, Universidad de los Llanos \\ hbastidas@unillanos.edu.co
}

Recibido 12 de Diciembre 2016, Aceptado 25 de Mayo 2017

\section{RESUMEN}

El cultivo de guanábana (Annona muricata L.) es uno de los más populares en Colombia, puesto que su fruto es muy consumido y requiere de buenos cuidados para su comercialización, en los últimos años se ha ido incrementando el área sembrada. La aplicación de productos químicos a estos árboles para la disminución de la enfermedad que los afecta, antracnosis (Colletotrichum gloeosporoides) es una constante; por lo tanto este proyecto investigó sobre otras alternativas probando productos orgánicos elaborados a base de extractos vegetales o biofertilizantes que puedan disminuir y controlar la incidencia y severidad de esta enfermedad, con el fin de reemplazar los agroquímicos que dejan residualidad y pueden ser perjudiciales para la salud humana. Para estas pruebas se establecieron los siguientes tratamientos: Mucuna pruriens (mucuna) (T1), Datura stramonium (borrachero) (T2) y un biofertilizante (T3) para comparar la severidad de la enfermedad contra un testigo (T0); se aplicó un diseño completamente al azar, cuatro tratamientos, tres replicaciones con tres unidades experimentales, se realizaron evaluaciones a los siete, doce y dieciocho días después de la aplicación de estos productos. El porcentaje de disminución con respecto a la severidad inicial que se tomó como cero (0), arroja que los productos que dieron un resultado positivo $(\mathrm{P}<0.05)$ en las aplicaciones de manera progresiva contra la enfermedad antracnosis fueron: el extracto con la planta 
Mucuna pruriens y el biofertilizante siendo de 78.35 Vs 18.36 y 50.81 Vs $20 \%$, respectivamente comparando la severidad inicial con la presentada a los 18 días después de la aplicación de productos. Se concluye que los mejores tratamientos fueron el de Mucuna pruriens seguido del biofertilizante.

Palabras clave: Annoneae, hongo, productos biológicos, frutas, biofertilizantes.

\begin{abstract}
The cultivation of soursop (Annona muricata L.) is one of the most popular in Colombia, since its fruit is very consumed and requires good care for its marketing, in recent years has been increasing the area sown. The applying of chemicals products to these tres for the reduction of the disease that affects them, anthracnose (Colletotrichum gloeosporioides) is a constant; therefore, this project investigated other alternatives testing organic products prepared from plant extracts or biofertilizer that can reduce and control the incidence and severity of this disease, in order to replace agrochemicals that leave residuality and can be detrimental to human health. For these tests the following treatments were established: Mucuna pruriens (T1), Datura stramonium (T2) and a biofertilizer (T3) to compare the severity of the disease against a control (T0); was applied a completely random design, four treatments, three replicates with three experimental units, the evaluations were carried out at seven, twelve and eighteen days after the application of these products. The percentage of decrease with respect to the initial severity which was taken as zero (0), shows that the products that gave a positive result $(\mathrm{P}<0.05)$ in applications progressively against anthracnose disease were: the Mucuna pruriens plant extract and the biofertilizer being 78.35 vs 18.36 and 50.81 vs $20 \%$ respectively, comparing the initial severity with that presented at 18 days after the application of products. It is concluded that the best treatments were the Mucuna pruriens followed by biofertilizer.
\end{abstract}

Keywords: Annoneae, fungus, biological products, fruits, biofertilizers. 


\section{RESUMO}

O cultivo de graviola (Annona muricata L.) é um dos mais populares na Colômbia, sendo que seu fruto é consumido e requer muito cuidado para sua comercialização, nos últimos anos tem aumentado a área plantada. A aplicação de produtos químicos para estas árvores para reduzir a doença que os afeta, antracnose (Colletotrichum gloeosporioides) é uma constante; portanto, esse projeto investigado em outras alternativas testar produtos orgánicos preparado a partir de extractos de plantas ou biofertilizantes que podem reduzir e controlar a incidência e gravidade da doença, a fim de substituir produtos químicos que deixam residuos e pode ser prejudicial para a saúde humana. Para estes testes os seguintes tratamentos foram estabelecidos: Mucuna pruriens (T1), Datura stramonium (T2) e um biofertilizante (T3) para comparar a gravidade da doença contra uma testemunha (T0); foi aplicado um desenho completamente aleatório,

quatro tratamentos, três repetições com três unidades experimentais, as avaliações foram realizadas aos sete, doze e dezoito días após a aplicação destes produtos. A percentagem de redução com respeito à gravidade inicial que foi tomado como zero (0), revela que os produtos que tiveram um resultado positivo $(\mathrm{P}<0.05)$ nas aplicações de maneira progressiva contra a doença antracnose foram: o extracto da planta Mucuna pruriens e biofertilizantes sendo $78.35 \mathrm{Vs}$ 18.36 e 50.81 vs $20 \%$, respectivamente, comparando a gravidade inicial com aquela apresentada em 18 dias após a aplicação dos produtos. Conclui-se que os melhores tratamentos foram de Mucuna pruriens followed by biofertilizer.

Palavras-chave: Annoneae, fungo, produtos biológicos, frutas, biofertilizantes.

\section{INTRODUCCIÓN}

En Colombia el cultivo de guanábana (Annona muricata L.) representa un renglón que ofrece magnificas oportunidades, en el mercado nacional e internacional (Cano et al, 2005; Márquez, 2009). Este árbol originario de la parte tropical de América casi siempre permanece verde (solo pierde las hojas al florecer), mide entre 3 a 7 metros de altura con crecimiento erecto, las hojas son alternas, 
simples, las cuales al estrujarse emiten un olor característico, el tronco es recto y de color grisáceo y ramifica a baja altura (FDA, 1993).

El cambio climático y el mal manejo agronómico han generado que las enfermedades aumenten en la guanábana, por lo tanto se buscan alternativas para el manejo de plagas y enfermedades que no impliquen la utilización de productos químicos; dentro de los problemas que más afecta el cultivo de guanábana y otros cultivos son las enfermedades foliares como la Antracnosis, el cual es producida por el hongo Colletotrichum gloeosporoides causante de grandes pérdidas (Acosta et al., 2001).

Para ello se busca una serie de alternativas en el control de la enfermedad mediante la implementación de buenas prácticas y ayudas al productor en la disminución de costos por control de la enfermedad; siendo la aplicación indiscriminada de pesticidas una práctica común desde hace muchos años utilizando productos de amplio espectro, por lo tanto se busca de manera alternativa la ayuda de otras plantas que generan un antagonismo con las enfermedades, especialmente Colletotrichum gloeosporoides, con la utilización de extractos vegetales, generando así prácticas de manejo de la enfermedad de manera preventiva y curativa (Álvarez et al., 2004).

Esta enfermedad es la más importante en la guanábana de los climas de humedad relativa alta $(\geq 75 \%)$ causando una pudrición negra en los frutos que ataca en todas las etapas de desarrollo, principalmente los tejidos tiernos, los frutos se momifican y caen (Figura 1), en el vivero provoca necrosis en el cuello del tallo y en ramas terminales (Andrades et al., 2009).

El control de esta enfermedad se realiza principalmente de manera química, utilizando productos de los grupos químicos: Benzimidazole, Ftalimidas ó Ditiocarbamato, que puede ser cada 10 días cuando el ataque de la enfermedad es severo o cada 30 días cuando la incidencia y severidad es baja (IIFT, 2011). 


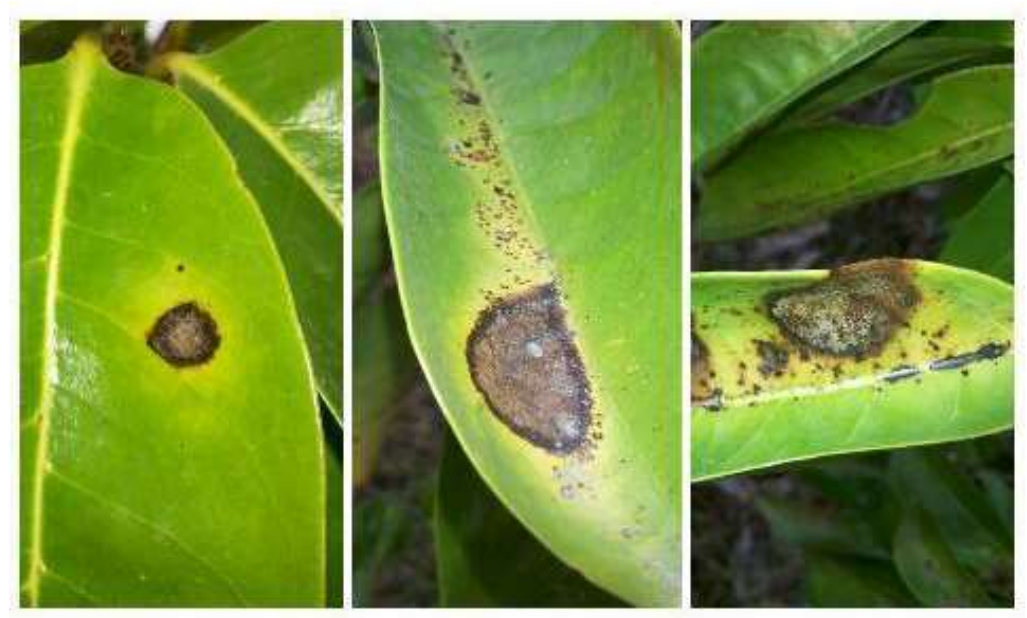

Figura 1. Enfermedad Antracnosis causado por el hongo Colletotrichum gloeosporoides en hojas de guanábana.

Fuente: Autores (2016).

Actualmente para el control de plagas, enfermedades y malezas en los cultivos se ha venido y utilizado organismos vivos, extractos de plantas y biofertilizantes, que al combinarlos con prácticas como la rotación de cultivos, alteraciones del $\mathrm{pH}$ del suelo con uso de enmiendas orgánicas, conformando así un control integrado preventivo, lo cual ha reducido el empleo de agroquímicos que dejan residuos tóxicos en los frutos y hojas que perjudican salud humana (FiBL, 2011).

Se ha comprobado que extractos y sustancias de algunas plantas tienen de actividad biológica para el control de arvenses, plagas y enfermedades en el sector agrícola; de esta forma, se pone en evidencia que la Mucuna pruriens (mucuna), y que además de poseer esas propiedades también se utiliza como cobertura en cultivos de palma de aceite, caucho, cítricos, forestales y frutales (Figura 2a) puesto que evita la erosión, controla arvenses, mantiene la humedad y su fertilidad porque fija importantes cantidades de nitrógeno atmosférico e incrementa la materia orgánica y su actividad biológica, lo cual conlleva al mejoramiento de la aireación y drenaje del mismo (AGROBROKERS, 2016).

Con la mucuna se elaboran compuestos nematicidas, que ayudados con la rotación de cultivos pueden reducir las poblaciones de estos parásitos, y también tiene efectos alelopáticos porque disminuye el crecimiento de malezas; otro uso para la mucuna es nutricional como forraje para animales por su alto contenido de 
nitrógeno en hojas (15-20\%). Las semillas tostadas de la mucuna se usan como un sustituto del café en Guatemala y México; en India, todas las partes de la planta tienen uso en más de 200 preparaciones medicinales indígenas; las semillas contienen hasta un $7 \%$ de Dopamina utilizado para el tratamiento de la enfermedad de Parkinson; en la medicina ayurvédica, su uso es para mejorar la lucidez mental y la coordinación muscular (Brunner et al., 2011; Hope et al., 2012).
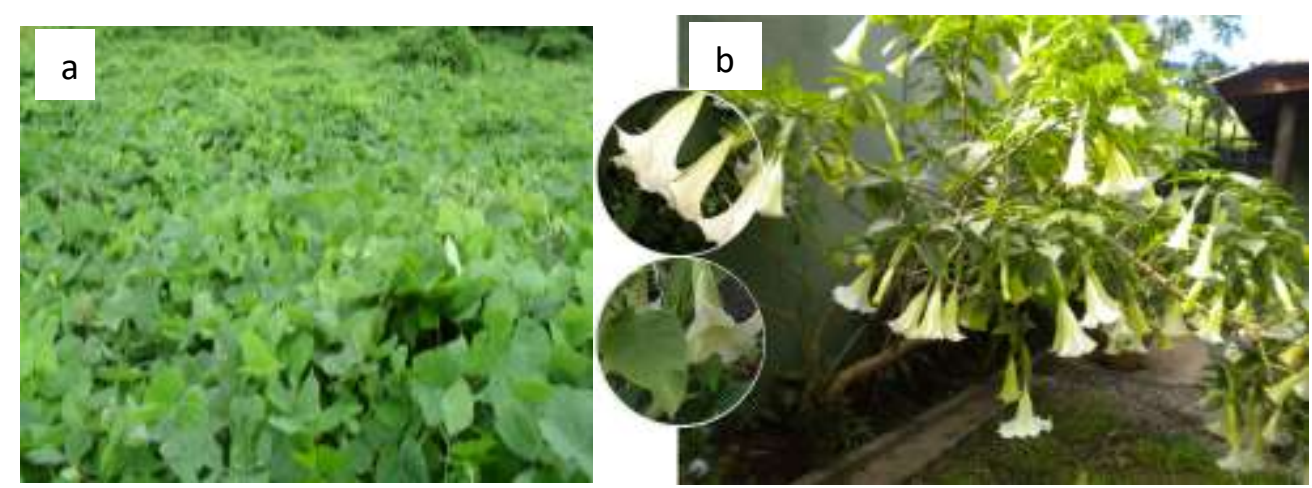

Figura 2. (a) Mucuna (Mucuna pruriens) y (b) Borrachero (Datura stramonium)

Fuente: Autores (2016)

Otra planta con componentes químicos para controlar plagas enfermedades es el borrachero o estramonio (Datura stramonium) (Figura 2b), que es considerada venenosa y pertenece a la familia de las solanáceas naturalizada en zonas templadas de todo el mundo. El género Datura contiene varias especies parecidas y polimorfas, todas ellas tóxicas de las cuales el estramonio es la más extendida, crece en zonas cálidas eutrofizadas como orillas de ríos, establos, estercoleros, escombreras y vertederos de basuras; es capaz de adaptarse a todo tipo de suelo, desarrollándose de manera más vigorosa en los húmedos con nitratos abundantes; no es consumida por el ganado, quizá por su desagradable olor, la planta contiene en todas sus partes gran cantidad de alcaloides ricos en atropina, escopolamina e hiosciamina, y los síntomas derivados de su consumo consisten en un síndrome anticolinérgico (Orozco, 2012).

Datura stramonium (Figura $2 \mathrm{~b}$ ) es una hierba anual de hasta 1.5-2 m. de altura, robusta y erguida, lampiña y ramificada con hojas entre ovadas y elípticas, apuntadas y ligeramente dentadas de 50-180 mm de longitud, florece desde Mayo hasta Octubre con flores blancas, tubulares, largamente embudadas, de hasta 10 
cm de longitud con cinco lóbulos cortos y pegados, que salen de forma aislada en el lugar de la ramificación; el cáliz es tubular y ligeramente inflado en la base, y los frutos son cápsulas ovoides de $35-70 \mathrm{~mm}$, generalmente cubiertas de espinas, esbeltas e iguales (Orozco, 2012).

Además de los extractos vegetales los biofertilizantes son otra alternativa natural para el control de plagas y enfermedades, existen algunos que contienen minerales a partir de extracto de cítricos, ricos en ácido ascórbico, tocoferoles, ácido grasos, monosacáridos y aminoácidos, lo cual hace que estos biofertilizantes tengan una excelente calidad nutricional, asegurando una aplicación efectiva y limpia sobre el tejido vegetal (Alarcón y Ferrara, 2000).

La aplicación de estos productos incide directamente en el proceso de defensa natural de la planta, pues contiene fucomarinas que son una potente fitoalexina, por otro lado, favorece el proceso de elicitación al activar la síntesis endógena generada por el ácido ascórbico; los bioflavonoides procedentes del extracto de cítricos, protegen de la oxidación al ácido ascórbico, potenciando su efecto. Ambos compuestos son antioxidantes, y actúan sinérgicamente preservando la calidad natural de frutas y hortalizas (Graeda et al., 2012).

El objetivo de este proyecto fue evaluar la aplicación de extracto vegetal Mucuna bracteata (mucuna) comparada con un biofertilizante y un testigo, para el control de la enfermedad foliar Antracnosis en Guanábana (Annona muricata L.) en la zona del Piedemonte llanero.

\section{METODOLOGÍA}

\section{Localización}

Este trabajo se realizó en Villavicencio, departamento del Meta, Colombia, zona que presenta características climatológicas de la siguiente manera: Coordenadas:(404'33.78" $\left.\mathrm{N} 73^{\circ} 34^{\prime} 49.50^{\prime \prime} \mathrm{O}\right)$, atura sobre el nivel del mar: 465 msnm, precipitación anual: $3250 \mathrm{~mm} / a n ̃ o$, humedad relativa: $75 \%$ y horas brillo solar anual: 4.5 horas (Acuuweather, 2016). 


\section{Cultivo}

Se sembraron 214 árboles de guanábana (Annona muricata L.) en un área de $9000 \mathrm{~m}^{2}$, donde estuvo asociada en sus primeros meses con plátano y maíz variedad guacavia; la distancia de siembra fue de siete metros entra surcos y seis entre plantas $(7 \times 6)$. Durante los dos primeros años, el manejo del cultivo se realizó de forma química, así como control de plagas y enfermedades; el mantenimiento fue realizado de manera mecánica por medio de guadaña evitando la existencia de hierbas arvenses o competidoras, y debido a este manejo el cultivo fue afectado por antracnosis ocasionada por el hongo Colletotrichum gloeosporoides, y para estimar su daño se contaron las hojas y tallos totales y luego los afectados por el hongo y se estableció un porcentaje de severidad de la enfermedad.

\section{Tratamientos}

Para evaluar los efectos de componentes naturales sobre la enfermedad antracnosis su usaron los siguientes tratamientos: (TO) testigo sin ninguna aplicación; (T1) utilizando extracto de mucuna: $30 \%$ de la planta + agua $70 \%$ ); (T2) extracto de borrachero: $30 \%$ de la planta + agua $70 \%$ y (T3) biofertilizante: producto $30 \%+$ agua $70 \%$.

Cada tratamiento elaborado con base vegetal se realizó respecto a 30\% hojas de la planta, es decir macerando sus tallos y hojas suculentas de forma que se obtuvo un extracto puro y $70 \%$ de agua, por ejemplo para obtener un litro de extracto vegetal para aplicar en campo se requirieron de $300 \mathrm{gr}$ de hojas y $700 \mathrm{ml}$ de agua, igual se hizo con el biofertilizante; estos componentes se licuaron, se pasaron por colador y se obtuvo el extracto vegetal y de biofertilizante listo para aplicar en campo. Los tratamientos se aplicaron en días soleados para evitar el lavada o pérdida del producto en el cultivo de guanábana que tenía dos años de sembrado y presentaba la enfermedad antracnosis; se humedeció totalmente el área foliar, para lo cual se utilizó una bomba de espalda, y para diferenciar los arboles tratados se colocó una bolsa plástica en alguna de sus ramas donde 
presentaba el follaje más afectado por la enfermedad; y se aplicaron dos litros de producto para nueve árboles.

\section{Diseño experimental}

Se utilizó un diseño completamente al azar, cuatro tratamientos, tres replicaciones

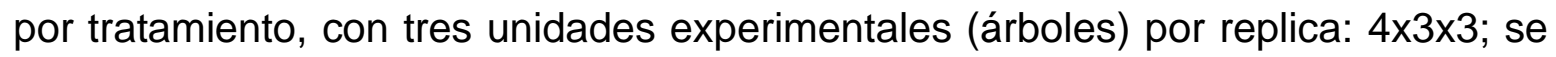
hicieron evaluaciones en tres diferentes fechas para observar su evolución. Se realizó análisis de varianza a la severidad de la enfermedad que fue determinada por el número de hojas y el área foliar afectada, y cuando hubo diferencias significativas $(p<0.05)$ se aplicaron pruebas de comparación de medias por el método de Duncan con un nivel de significancia del $5 \%$.

Antes de aplicar los productos en cada replicación, se realizó una evaluación de incidencia y severidad, buscando el porcentaje de afectación inicial en los árboles de guanábana (Tabla 1), luego de la aplicación de los componentes naturales se hicieron evaluaciones a los 7 y 12 días después, determinando así el porcentaje de afectación de la enfermedad en cada tratamiento, para observar su respuesta a los mismos, y por ultimo una evaluación a los 18 días, para establecer el mayor y/o menor efecto de los tratamientos.

Tabla 1. Severidad inicial de los arboles antes de aplicar su respectivo tratamiento (\%)

\begin{tabular}{cc}
\hline TRATAMIENTO & VALOR \\
\hline Testigo (Sin ninguna aplicación) & 18.36 \\
Mucuna pruriens (Mucuna) 30: 70 de agua & 20.00 \\
Datura stramonium (Borrachero) 30: 70 de agua & 20.00 \\
Biofertilizante 30: 70 de agua & 20.33 \\
\hline
\end{tabular}

\section{RESULTADOS Y DISCUSIÓN}

Se puede interpretar que en las primeras evaluaciones de las tres repeticiones se encontró un efecto positivo en el tratamiento elaborado con la planta Mucuna pruriens disminuyendo la incidencia y severidad de la enfermedad en los árboles 
de guanábana, seguido del biofertilizante que también mostró una acción positiva en el control de la enfermedad (Tabla 2).

Tabla 2. Severidad de antracnosis (Colletotrichum gloeosporoides) a los siete días después de la aplicación de los productos (\%)

TRATAMIENTO

Testigo (Sin ninguna aplicación)

Mucuna pruriens (Mucuna) en proporción 30: 70 de agua

Datura stramonium (Borrachero) 30: 70 de agua

Biofertilizante en proporción 30: 70 de agua
VALORES

$21.67^{\mathrm{b}}$

$6.67^{\mathrm{a}}$

$24.00^{\mathrm{b}}$

$17.80^{\mathrm{b}}$

Letras distintas indican diferencias significativas $(P<0.05)$

La segunda evaluación, después de 12 días de aplicación de los productos, se siguió observando que mucuna tiene un efecto positivo controlando la enfermedad $(\mathrm{P}<0.05)$, puesto que no permitió que la misma progrese, deteniendo el desarrollo del hongo, seguidamente el producto biológico sigue disminuyendo la incidencia de la enfermedad, mientras que en el testigo se incrementó la afección (Gráfica 1).

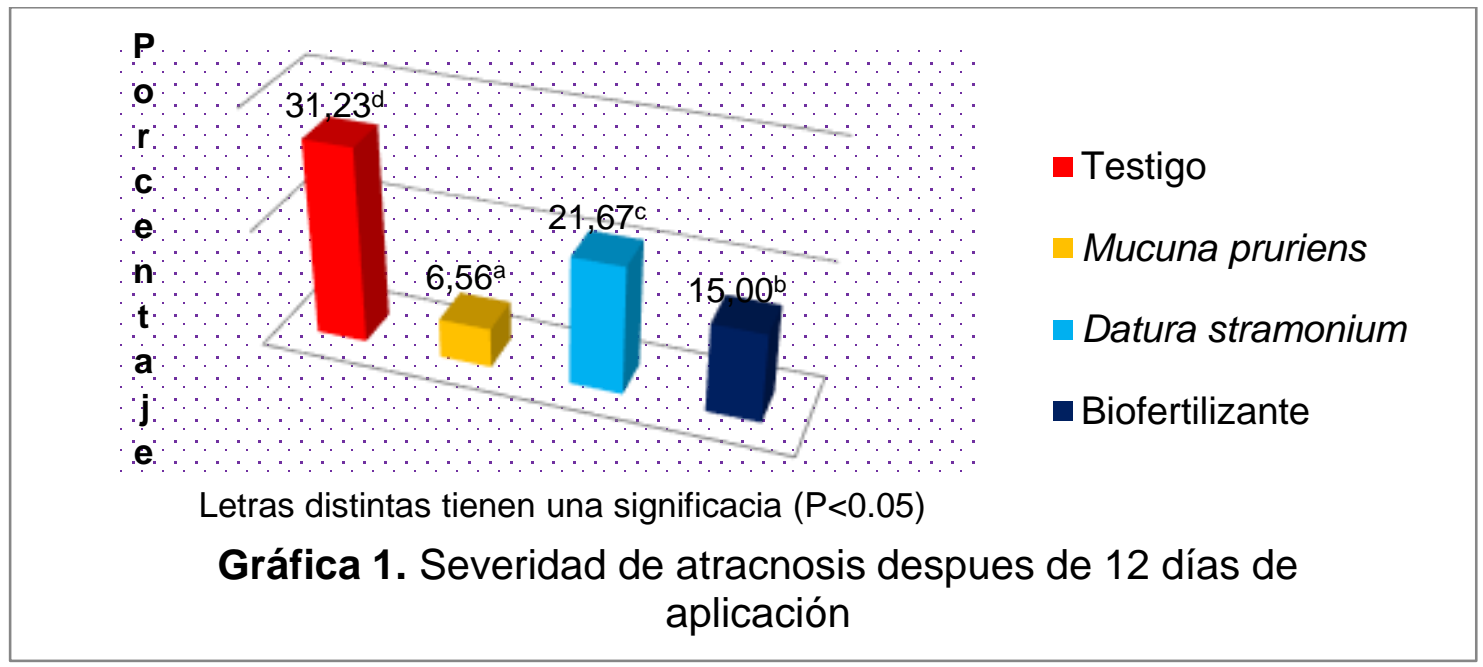

En la tercera evaluación a los 18 días post-aplicación, se observa que el tratamiento a base de hojas de mucuna arroja un mejor resultado $(\mathrm{P}<0.05)$ con respecto a la disminución de la enfermedad, seguidamente no en igual proporción pero si positivamente el biofertilizante, el testigo continuó siendo el más afectado por la enfermedad y el tratamiento con Datura stramonium (borrachero) no fue efectivo (Grafico 2). 


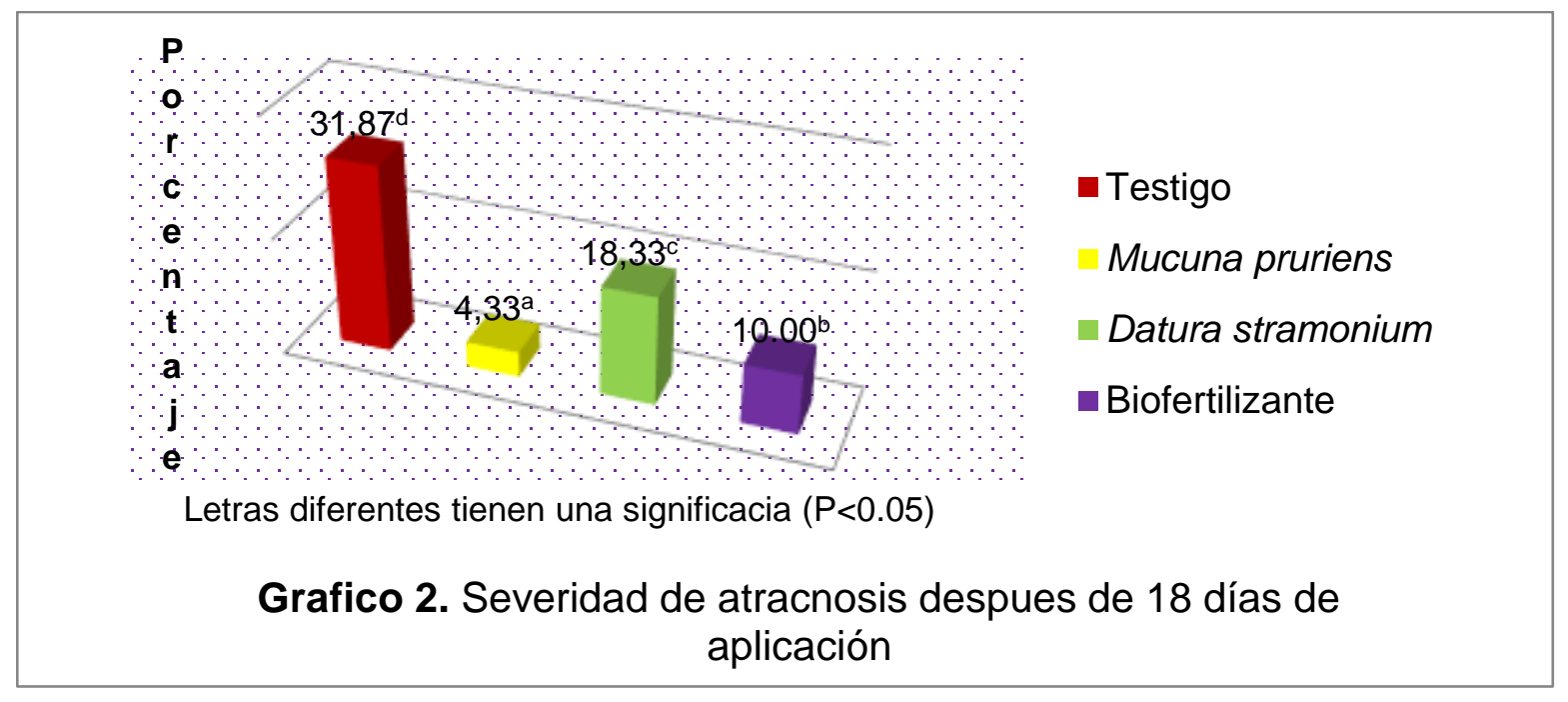

El porcentaje de disminución con respecto a la severidad inicial que se tomó como cero (o), indica que los productos que arrojaron un resultado positivo $(P<0.05)$ en las aplicaciones de manera progresiva contra la enfermedad antracnosis fueron: el extracto con la planta Mucuna pruriens y el biofertilizante siendo de 78.35 Vs 18.36 y 50.81 Vs $20 \%$ respectivamente, comparando la severidad inicial con la presentada a los 18 días después de la aplicación de tratamientos (Tabla 2 y Gráfica 3). Con el extracto de borrachero se controló poco la severidad de la enfermedad $23.63 \%$, mientras que en el testigo se incrementó el daño en un $73.58 \%$, observando un desmejoramiento de las hojas y tallos de la planta de guanábana (Gráfica 3). De acuerdo a estos resultados, la mucuna demostró una gran eficiencia en el control de la antracnosis, lo cual se debe a su contenido de sustancias naturales generadas por su metabolismo secundario, como un mecanismo de defensa ante el ataque de hongos, bacterias, insectos y pájaros, siendo estos componentes: L-Dopa, fenoles, taninos, inhibidores de proteasas, lectinas y ácido fítico (Janardhanan et al., 2003; Adebowale et al., 2005). Se ha comprobado que L-Dopa produce desequilibrio celular, por lo tanto es muy factible que haga este efecto sobre el hongo Colletotrichum gloeosporoides; además con respecto al contenido de fenoles y taninos en la mucuna Vadivel y Janardhanan, (2005) señalan que los primeros son considerados como sustancias corrosivas y pueden quemar las membranas celulares, e igualmente los segundas alteran el funcionamiento celular, daños que se pueden reflejar en el hongo. 


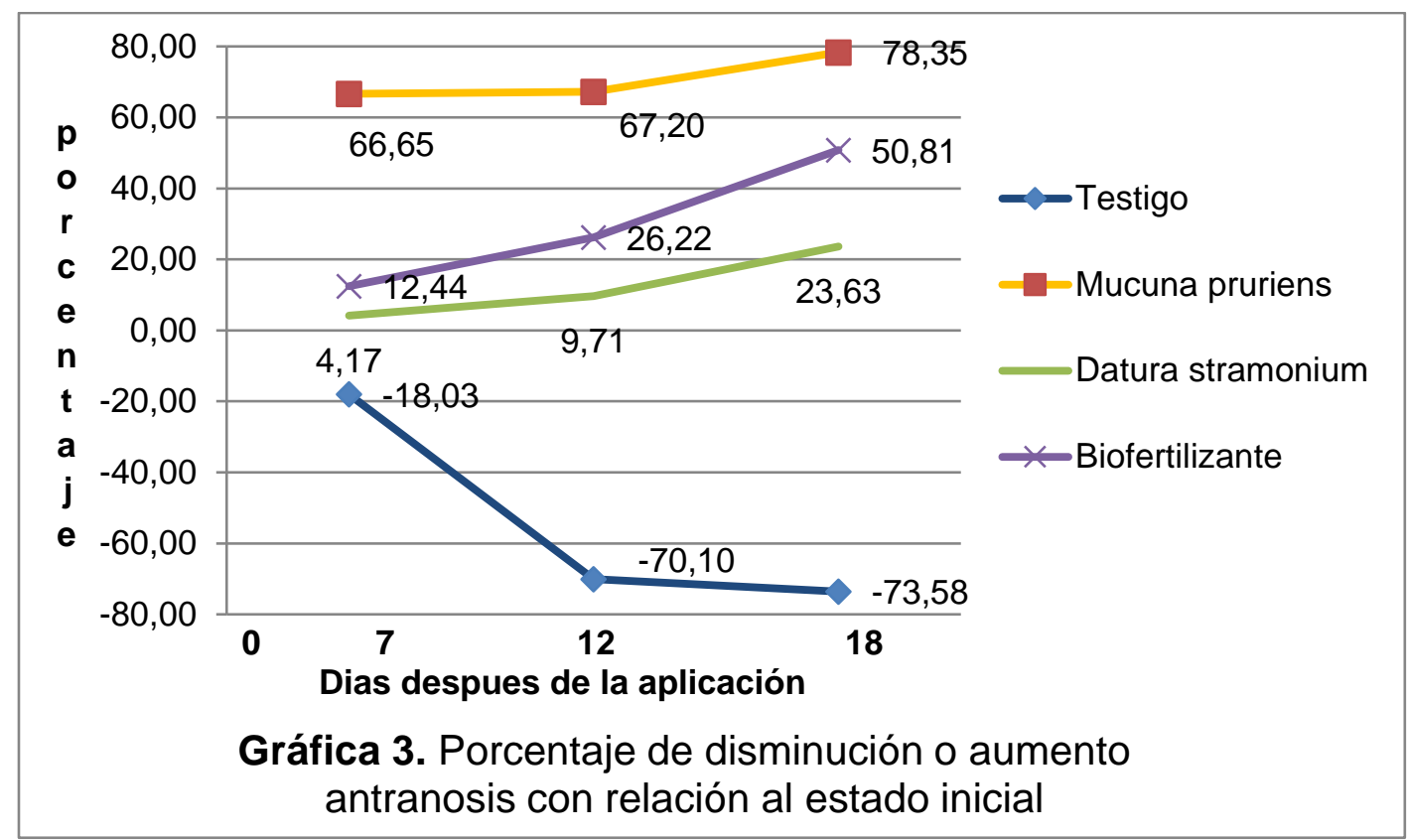

El tratamiento elaborado a base de hojas de mucuna demostró una disminución de $86.1 \%$ de la enfermedad de antracnosis en los arboles tratados, a los 18 días después de su aplicación con respecto al testigo $(\mathrm{P}<0.05)$, el biofertilizante presentó también resultados positivos $(P<0.05)$, demostró tener mayor efecto que el borrachero, siendo superior su acción sobre la enfermedad al testigo a los 18 días en 68.0 y $41.3 \%$ respectivamente (Gráfica 4).

Se puede analizar que entre los productos que mejores resultados mostraron en los tres periodos de evaluación fue el extracto vegetal elaborado a base de hojas de mucuna y el biofertilizante presentando porcentajes superiores al $12 \%$ en la disminución de la enfermedad y durante cada evaluación, estos valores aumentaron de manera progresiva, a medida que avanzó el tiempo demostrándose un mayor control de la enfermedad, aunque entre los dos productos realizaron un buen control, el extracto vegetal fue el que obtuvo un mayor porcentaje en la disminución del ataque de la enfermedad, con altas posibilidades de no dejar residualidad. 


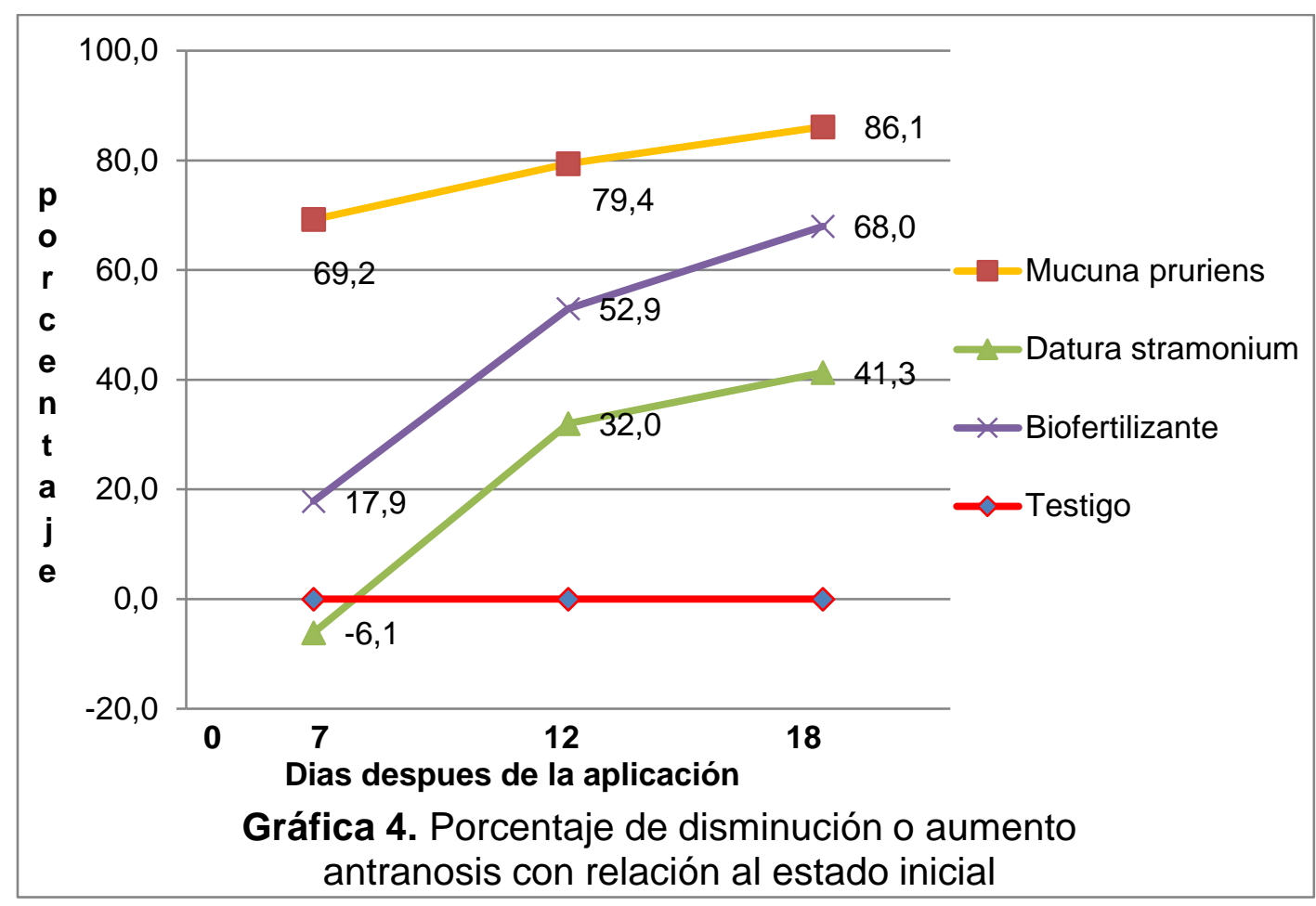

Con relación al biofertilizante, aunque no es un fungicida si es un abono foliar, por lo cual se puede llegar a determinar que el aporte de nutrientes a la planta genera un balance nutricional positivo, haciendo que el árbol adquiera mayor resistencia y rusticidad frente a la enfermedad.

\section{CONCLUSIONES}

En base a las diferentes pruebas realizadas con los extractos vegetales se puede concluir que el mejor producto para el control del hongo Colletotrichum gloeosporoides, causante de la enfermedad antracnosis, que es de importancia económica en el cultivo de guanábana, es el elaborado con hojas de Mucuna pruriens, puesto que las plantas tratadas con este extracto obtuvieron una disminución de la enfermedad del $86.1 \%$ con relación a la severidad inicial, en un periodo de 18 días posteriores a su aplicación.

Otro producto que generó una disminución considerable $(50.81 \%)$ de la enfermedad con relación a la severidad inicial fue el biofertilizante, que aunque no es un fungicida, mostró un efecto positivo que se incrementó a medida que pasaro 
el tiempo, deduciendo que su efecto en la planta se basa en la asimilación de los nutrientes aportados.

Los productos aplicados para el control de la antracnosis no ocasionaron daño a las hojas y tallos de planta, al contrario se observó mejoramiento de su aspecto en menor o mayor dependiendo de tratamiento, debido al control del hongo Colletotrichum gloeosporoide y con ello la disminución de sus efectos patológicos.

\section{REFERENCIAS BIBLIOGRÁFICAS}

1. Acosta RM, Nieto ÁD, Domínguez ÁJ, Delgadillo SF. Calidad y tolerancia en frutos de papaya (Carica papaya L.) a la inoculación del hongo Colletotrichum gloeosporioides Penz., en postcosecha. Revista Chapingo Serie Horticultura, 7 (1): 119-124. 2001.

2. ACUUWEATHER. Colombia tiempo y meteorología. 2016. Recuperado 30 Mayo 2016.2 Disponible En: http://www.accuweather.com/es/co/villavicencio/105930/weatherforecast $/ 105930$

3. Adebowale YA, Adeyemi A, Oshodi AA. Variability in the physicochemical, nutritional and antinutritional attributes of six Mucuna species. Food Chemistry, 89 (1): 37-48. 2005.

4. AGROBROKERS. Mucuna Bracteata, 2016. Recuperado 17 Mayo 2016. Disponible En: http://agrobrokers.com.co/sitio/

5. Alarcón A, Ferrara, R. Biofertilizantes: importancia y utilización en la agricultura. Agricultura Técnica en México, 26 (2):191-203. 2000.

6. Álvarez E, Ospina CA, Mejía JF, Llano GA. Caracterización morfológica, patogénica y genética del agente causal de la antracnosis (Colletotrichum gloeosporioides) en guanábana (Annona muricata) en el Valle del Cauca. Fitopatología Colombiana, 28 (1): 1-8. 2004.

7. Andrades I, Yender F, Labarca J, Ulacio D, Paredes C, Marin Y. Evaluación de antracnosis (Colletotrichum sp.) en guanabana (Annona muricata L.) tipo gigante en el sector moralito del estado Zulia, Venezuela. Revista Científica UDO Agrícola, 9 (1): 148-157. 2008.

8. Brunner B, Beaver J, Flores L. Hoja informativa Mucuna. Proyecto de Agricultura Orgánica Z-NRCS-007. Departamento de Cultivos y Ciencias Agroambientales, Estación Experimental Agrícola de Lajas, Puerto Rico. 5 p. 2011 Recuperado 17 de Mayo 2016. Disponible En: http://prorganico.info/mucuna.pdf

9. Cano CM, Stringheta PC, Ramos AM, Cal VJ. Effects of the carriers on the microstructure of mango powder obtained by spray drying and its functional characterization. Innovative Food Science \& Emerging Technologies, 6 (4): 420-428. 2005.

10. Fundación de Desarrollo Agropecuario (FDA). El cultivo de la guanábana. Santo Domingo, República Dominicana. Boletín Técnico N. 12: 2-4. 1993. 
11. Grageda O, Díaz A, Peña J, Vera J. Impacto de los biofertilizantes en la agricultura. Revista Mexicana Ciencias Agrícolas, 3 (6): 1261-1274. 2012.

12. Hope NS, Ogueli GI, Cortelazzo A, Cerutti H, Cito A, Aguiyi JC, Guerranti R. Effects of Mucuna pruriens protease inhibitors on Echis carinatus venom. Phytotherapy Research, 26 (12): 1913-1919. 2012.

13. Instituto de Investigaciones de Fruticultura Tropical (IIFT). Instructivo técnico para el cultivo de guanábana. Editorial Vento. Asociación Cubana de Técnicos Agrícolas y Forestales, Cuba, p 1-4. 2011

14. Janardhanan K, Gurumoorthi P, Pugalenthi M. Nutritional potential of five accessions of a South Indian tribal pulse, Mucuna pruriens var. utilis I. The effect of processing methods on the content of L-Dopa, phytic acid and oligosaccharides. Tropical and Subtropical Agroecosystems, 1 (2-3): 141-152. 2003.

15. Orozco, B. Estramonio (Datura stramonium). 2012. Recuperado 18 Mayo $2016 . \quad$ Disponible En: https://1276orozko.wordpress.com/2012/09/18/estramonio-datura-stramoniumorozko-bizkaia/

16. Research Institute of Organic Agriculture (FiBL). African Organic Agriculture Training Manual- Pest, Disease and Weeds. Version 1.0 June. Ed. Weidmann Gilles, Kilcher, L. Frick, Switzerland. 14 p. 2011.

17. Márquez CJ. Caracterización fisiológica, físico-química, reológica, nutraceútica, estructural y sensorial de la guanábana (Annona muricata L. cv. Elita). Tesis de Doctorado, Universidad Nacional de Colombia, Facultad de Ciencias Agropecuarias. Medellín, Colombia. 2009.

18. Vadivel V, Janardhanan K. Nutritional and antinutritional characteristics of seven South Indian wild legumes. Plant Foods for human Nutrition, 60 (2): 6975. 2005. 